\title{
Article \\ A Probabilistic Approach to Small Fatigue Crack Initiation and Sizing Using Acoustic Emission
}

\author{
Azadeh Keshtgar * and Mohammad Modarres \\ Center for Risk and Reliability, University of Maryland, College Park, MD 20742, USA; modarres@umd.edu \\ * Correspondence: azadeh.keshtgar@gmail.com or keshtgar@umd.edu
}

\begin{abstract}
One of the major concerns in structural health management (SHM) is the early detection of a growing crack. Using this, future damage due to crack propagation can be mitigated or eliminated by implementing proper repair and maintenance. Acoustic Emission (AE) is a non-destructive testing (NDT) method with potential applications for locating and monitoring fatigue cracks. The research presented in this paper focuses on SHM using AE. A novel AE signal analysis approach was proposed in order to detect crack initiation and assess small crack lengths. Experimental investigation indicated that initiation of a crack could be identified through the statistical analysis of the resulting features of the AE signals. A probabilistic AE-based model for small fatigue crack sizing was developed and the uncertainties of the model were estimated. In addition, a probabilistic model validation approach was implemented to confirm accurate estimation of the results. The outcome of this research can be used to evaluate the integrity of structures under fatigue loading. The proposed approach can also be applied as an approach to manage health and assess prognosis of structures.
\end{abstract}

Keywords: acoustic emission; crack initiation; small crack growth; structural integrity; bayesian estimation

\section{Introduction}

In materials engineering, fatigue cracking occurs when a material is subjected to cyclic loading. Structures such as bridges and airframes can be subjected to wide ranges of loading conditions during their operations. Extreme repeated loads may cause initiation of cracks and crack growth during the life of a structure. Therefore, a crack initiation and small crack growth assessment approach would be needed to provide detection and prognosis of cracks in critical structures under fatigue loadings. This approach ensures that fatigue cracks would not propagate and cause catastrophic failures.

Several researchers have focused on the study of small crack growth behavior and different small-crack test methods [1-4]; but no AE-based model for small crack growth have been offered. However, most of the success in correlating AE activities with crack growth has involved the latter stages of crack growth (Regions II and III of the crack growth curve) [5-7].

Few studies have been carried out to combine fracture toughness experiments with $\mathrm{AE}$ techniques in order to detect the damage initiation. Marquez and Olivares [8] utilized an AE recording system to determine crack initiation and propagation at a thermally sprayed coating interface of nickel-chrome alloy with substrate of AISI 1045 steel. This study initiated an idea of relating abnormal behavior of AE signals to crack related events. However, it was not until 2005 that Chaswal [9] identified that AE amplitude is about an order of magnitude lower in Region $I$ than in Region II due to lower $\Delta K$ values. They investigated how low amplitude bursts in a short duration of time in Region $I$ correspond to micro-cleavage in thermally aged steel plates. Rahman, et al. [10] and Mba [11], hypothesized that a sudden and significant increase in AE events corresponds to damage initiation. Rahman [10] offered that significant increase in hit count data might correspond to incipient damage due to wear in rolling elements during their contacts. Mba [11] presented result of their experimental investigation for detecting natural crack in slow speed shafts. 
More recent studies [12-15] reported that much of the AE activity is recorded in the initial stages of damage accumulation compared to the later stages, and attribute this to material inclusions that crack in the early degradation periods. These studies hypothesize crack propagation rate based on the AE signals, but do not directly correlate them to crack initiation, nor do they consider associated crack detection probability and crack sizing errors using the detected $\mathrm{AE}$ features.

Despite a number of studies related to AE-based detection of fatigue damage in the literature, there has been no integrated approach for detection and sizing of crack initiation. Moreover, application of AE-based techniques for in-situ monitoring of small crack growth and initiation has not been adequately developed. Additionally, AE-based probabilistic prediction method for small crack initiation and growth is absent in the literature.

In this paper a new methodology of statistical AE data analysis for detecting crack initiation and developing a probabilistic AE model of small crack growth is introduced. Also, a relationship between small crack length and AE signal features generated during small crack growth is developed. The experimental setup and procedure used in fatigue testing is also explained in detail. Moreover, the procedure of probabilistic model development and validation is discussed and the uncertainties of the model are assessed. Figure 1 illustrates an overview of the proposed methodology and shows that post-processing of the captured data in conjunction with model error estimation results in establishing a probabilistic AE-based model. The developed model can be used for detection of crack initiation as well as assessment of small crack growth behavior. This method can be used to assess the reliability and evaluate health of the structures by estimating the probability of structural failure at a specified number of applied load cycles, including associated uncertainties.

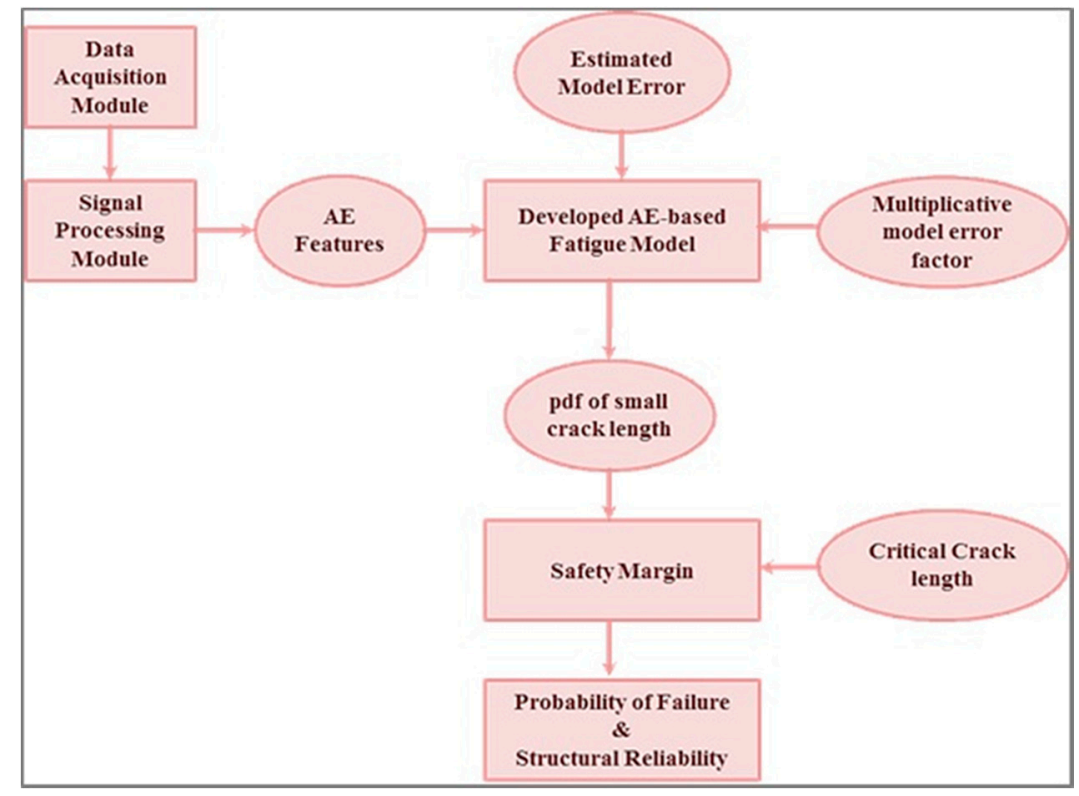

Figure 1.Methodology Overview

\subsection{The Basic Theory of AE}

Acoustic emission is defined as a transient elastic wave generated by the rapid release of energy within a material and an AE signal is the electrical signal produced by a sensor in response to this wave $[16,17]$. The use of AE has been primarily limited to statistical analysis of AE hits. An AE hit is defined as one acoustical waveform and the result of located hit is referred to as an AE event. The waveform has traditionally been described in terms of its features. An example of a typical AE waveform and its features is shown in Figure 2. In addition to recording the number of acoustic hits and correlating this number to the level of damage [18], it is also possible to record certain features of the AE waveforms. Some features are defined with respect to the specified threshold limit. These waveform features include but not limited to the ones listed below [19, 20]: 
- Counts: Also known as "ring down count" is defined as the number of times that the AE signal amplitude exceeds a predefined subjective threshold value. In general, very frequent larger than threshold signal amplitudes produce high counts. The AE count feature provides a measure of strength of the AE hit.

- Amplitude: The AE amplitude is the largest voltage peak in the waveform signal. Peak amplitude usually recorded in log units (decibels) to provide accurate assessment for both large and small AE hits.

- Energy: AE energy is the measured area under the rectified signal envelope. To measure energy directly, the waveform signal needs to be digitized and integrated (Analog-to-digital convertor).

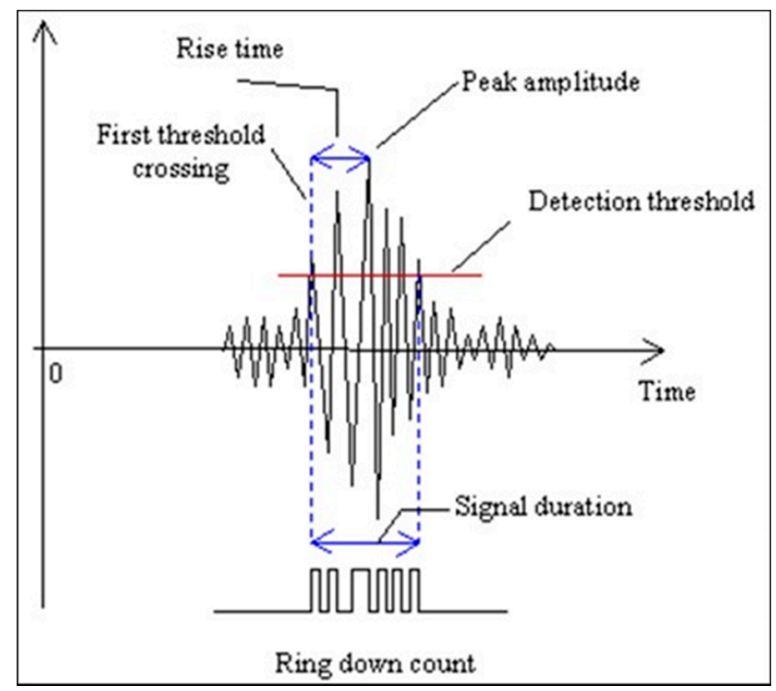

Figure 2. A typical AE signal and its characteristics [21]

\subsection{Fatigue Crack Initiation and Small Crack}

There is no universally accepted definition for fatigue-induced "small crack" and "crack initiation". Most experts consider cracks less than 1mm in length $(a<0.001 \mathrm{~m})$ as small [22], [23]. Fatigue crack initiation is a subjective notion as well. Some consider crack initiation as corresponding with a fatigue phenomenon and some associate it with an arbitrarily specified crack length. For example, the U.S. Navy defines the presence of a crack $250 \mu \mathrm{m}$ in length, as the point where crack initiation occurs [24], [25]. Furthermore, others consider a crack length ranging from a size of grain diameter to about $100 \mu \mathrm{m}$ as crack initiation length depending on material and scale of interest. However, ranges of values have been used for identification of crack initiation in different materials within the literature (e.g., $51 \mu \mathrm{m}$ for carbon steel, $120 \mu \mathrm{m}$ for BS250A53 steel and $1 \mathrm{~mm}$ for En7A steel [26] and up to $500 \mu \mathrm{m}$ for aluminum [27]).

A multi-stage fatigue approach developed at the Mississippi State University [28] divided the fatigue life of structure into three different stages of incubation, small crack growth and large crack growth. Based on this work, a SHM study by Papazian, et al. [24] defined a physically small crack to be between the incubated size (starting about $2.8 \mu \mathrm{m}$ ) and a crack size that is treatable by the linear elastic fracture mechanics (LEFM) models, which is about $250 \mu \mathrm{m}$ for AL7056-T651.

In this paper, a subjective fatigue crack initiation length was used for Al7075-T6. This selection was primarily made based on the limitations in the crack size measurement method (i.e., optical microscopy) used in this study rather than a specific fatigue phenomenon. As such this study uses a subjective crack length of $50 \mu \mathrm{m}$ as the point of crack initiation. It is determined, however, that this crack initiation length is about a quarter of the average grain diameter for Al7075. It has been shown that approximately a 1- $\mathrm{mm}^{3}$ of the above-mentioned material contains 700 grains [24]. Assuming spherical geometry of grains, the volume of each grain is about $1.43 \times 10-3 \mathrm{~mm}^{3}$ and the diameter of each grain can be estimated as $0.140 \mathrm{~mm}(140 \mu \mathrm{m})$. Moreover, the applied optical crack measurement method carries some limitations for sizing of smaller cracks. 


\section{Experimental Setup}

To study the relationship between small crack length and the resulting AE signal, a group of standard experiments was performed under a controlled loading condition. In this section experimental procedures including specimen selection, test set up, fatigue testing, crack length measurement and $\mathrm{AE}$ data acquisition are presented.

\subsection{Specimen and AE Instrumentation}

Among various test techniques that have been used to record the growth of small fatigue cracks, only a few can provide useful measurements of small-crack growth [1], [22]. Some measurement methods involve stopping the test to observe and measure the length of small crack. Obviously, these methods provide post-test information, making real-time monitoring of the small crack behavior impossible. It was desirable to not only measure crack length and crack growth rate of small fatigue cracks, but to do so in real time in order to correlate the state of damage to AE signal properties.

Since in this research the observation of small crack length was done through in-situ optical microscopy, a series of standard flat dog-bone test specimens with the capability of being used for small crack monitoring were developed based on the ASTM standard [29]. The samples of Aluminum alloy 7075-T6 tested were inspected for any visible defects and assumed to be free of any fabrication defects. Figure 3 is a schematic and geometric specification of the standard flat dog-bone specimen used in this research. Note that the edge notch is a semi-circle with the radius of $0.5 \mathrm{~mm}$.

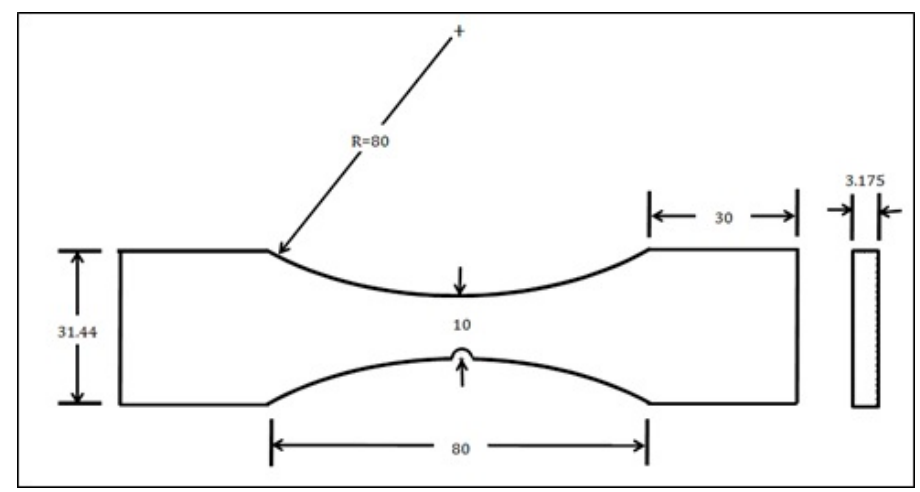

Figure 3. Drawing of flat dogbone samples (all dimensions in $\mathrm{mm}$ )

An advanced DiSP-4 AE system was used to record the AE signals resulting from the propagation of the crack inside the material. This monitoring system consisted of four main parts: a single wide band differential AE sensor to collect the signals, an amplifier to amplify the resulting signals, a data acquisition module to perform primary filtration and record the signals, and a software module (AEWin) to display the data and perform feature extraction. AEWin software was used for data acquisition, real time simultaneous $\mathrm{AE}$ feature, waveform processing, displaying and fast storage. The DiSP-4 hardware system is controlled via the AEWin software. The sensor is made of stainless steel case with a ceramic face with the dimension of $0.7^{\prime \prime} \mathrm{OD} \times 0.65^{\prime \prime} H$. The sensor operating frequency range is from 125 to $1000 \mathrm{kHz}$ and its peak sensitivity varies between -61 and $56 \mathrm{~dB}$.

\subsection{Optical Microscopy}

Fatigue cracks were monitored by direct measurement using an optical microscope on the front surface of the samples. The optical measurement system is comprised of several components: a high magnification optical microscope, a video camera attached to the microscope that records the crack growth path for the duration of the fatigue test, a dual arm fiber optic illuminator, a high resolution monitor, an image processing software with the time-lapse photography capability, and a micro-meter scale to calibrate the photographs taken. This measurement system allows for detection 
of small crack lengths, and is sufficient for capturing enough data to correlate observed crack length with the AE signals. Figure 4 shows the optical microscopy test set up used for the small crack experiment.

The assembled optical microscopy unit was focused on the specimen using 100X magnification to monitor the specimen notch edge, from which the crack was expected to initiate. The length of practically detected cracks varies depending of the degree of specimen's minute shaking induced by the load frame operation and consequently the ability of the microscope to focus on the notch. The smallest crack length practically measurable at $100 \%$ of times for all the experiments found to be 0.05 $m m(50 \mu \mathrm{m})$. Cracks as small as $20 \mu \mathrm{m}$ was detected at some cases, but a crack of $50 \mu \mathrm{m}$ length was detectable at all times regardless of the specimen vibration and optical focus. Crack length was monitored until it exceeded $0.25 \mathrm{~mm}(250 \mu \mathrm{m})$. At this point, sufficient small crack data was collected and crack measurement was terminated.

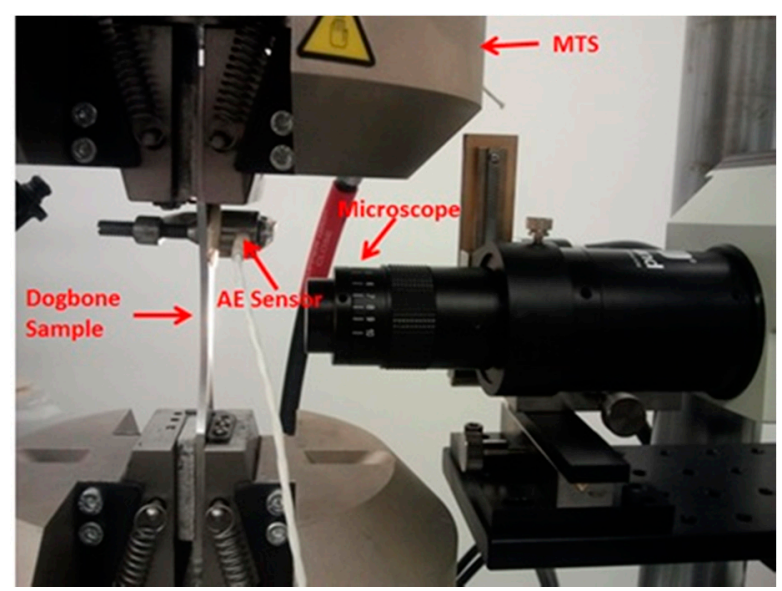

Figure 4. Test set up

\subsection{Fatigue Testing}

In the experimental set up described above, eight fatigue specimens were tested at different loading conditions using both AE and optical microscopy measurement. Table 1 lists details of loading parameters for the tests performed.

\begin{tabular}{c|ccc}
\multicolumn{5}{c}{ Table 1. Details of loading parameters for all experiments. } \\
\hline $\begin{array}{c}\text { Test } \\
\text { Reference }\end{array}$ & $\begin{array}{c}\text { Loading Frequency } \\
\text { (Hz) }\end{array}$ & $\begin{array}{c}\text { Loading Ratio } \\
(R)\end{array}$ & $\begin{array}{c}\text { Force (min-max). } \\
\text { KV }\end{array}$ \\
\hline T1 & 3 & 0.1 & $0.8-8$ \\
T2 & 3 & 0.08 & $0.64-8$ \\
T3 & 3 & 0.1 & $0.8-8$ \\
T4 & 2 & 0.5 & $6.5-13$ \\
T5 & 3 & 0.1 & $0.8-8$ \\
T6 & 2 & 0.3 & $3-10$ \\
T7 & 2 & 0.5 & $6.5-13$ \\
T8 & 2 & 0.3 & $3-10$ \\
\hline
\end{tabular}

\section{Data Analysis and Test Results}

Acoustic Emission signals may be generated from a number of possible sources including background noise, micro-crack generation, or plastic deformation. In order to reduce uncertainties and determine the AE signals corresponding to crack growth, applying noise reduction techniques on the captured data is required. Noise reduction approaches are discussed in the following section. 


\subsection{Noise Reduction}

Various de-noising techniques have been proposed to filter AE signals due to crack growth [27-29]. In the first step, the recorded AE data was filtered using the DiSP-4 source location software. The filtration was done using a band pass filter $(200 \mathrm{kHz}-3 \mathrm{MHz})$ to eliminate emissions from extraneous sources.

A detection threshold needs to be determined to filter the background noise. A balance must be made between setting event threshold limit high enough to filter out the majority of the background noise, yet low enough to pick up the beginning of the crack growth. Using a dummy specimen the threshold value was identified to be at $35 \mathrm{~dB}$ which was above operational background noise of the machine. This threshold allows for better capturing of crack-related signals.

It has also been observed that $\mathrm{AE}$ events occurring during the loading portion of a cycle are related to crack growth $[27,30,31]$. Therefore, the AE data taken during the loading portion of each cycle were used for data analysis. In addition, majority of researchers have assumed that only events occurring close to the maximum or peak load are associated directly with crack growth [27, 28, 32]. So, the filtered AE events were separated for different percentages of the applied load range and it was determined that the AE events occurring within the top $20 \%$ of peak load shows the closest correlation with crack lengths [6].

Furthermore, the initial cycles of the recorded data that represent the transient onset response were cut out and the analysis was done using the remaining data. This transient onset response is just the noise of the system at the start of the test and does not carry useful information.

\subsection{Crack Measurement}

Eight fatigue specimens under constant amplitude loading were monitored using both AE and optical microscopy. The optical microscopy system with time-lapse photography was used for monitoring crack growth. The lengths of pictured cracks were measured using the Java-based image-processing software. Crack measurement was started before $50 \mu \mathrm{m}$ and continued until the crack length larger than $250 \mu \mathrm{m}$ was observed. At this length the crack exceeds the Navy definition of crack initiation [25] and the crack measurement was terminated. Figure 5 illustrates an example of a sequence of images captured during crack growth of experiment (T1).

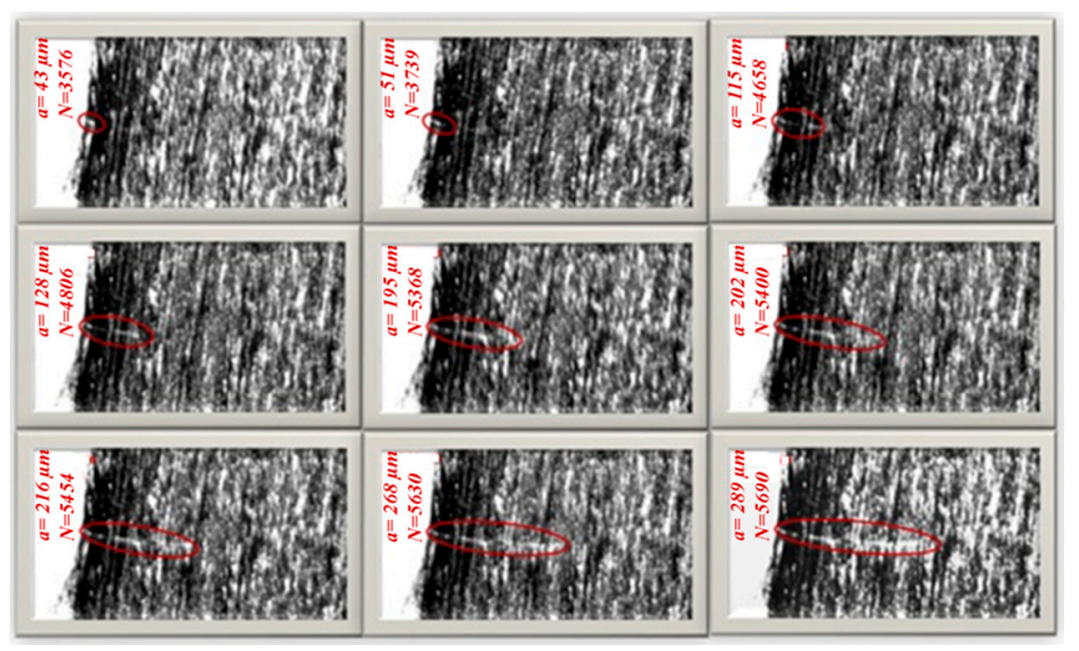

Figure 5. A sequence of crack growth images including the associated crack length (a) and loading cycle $N$.

\subsection{Experimental Uncertainties and Errors}

Crack measurement data may be uncertain in nature due to detection uncertainties and measurement errors associated with the optical measurement and sizing process. Estimation of crack lengths and consequently AE-model prediction can be affected by these uncertainties. 
Therefore, the uncertainties should be characterized properly and should be considered in determining the true crack length for AE model development. In this section, experimental crack length measurement error is discussed and later used to quantify the uncertainty and validity of the developed AE model.

\subsubsection{Probability of Detection (POD)}

Probability of detection expresses the probability of detecting a crack of a given length and is a common metric to assess the capability of a detection technique. A crack of a given length might be optically detected only at certain percentage of the time (out of the total number of tests) depending on factors such as sample subsurface cracks, specimen vibration, cyclic loadings, optical focus and human error. During the experiments, sometimes a crack was too small to be detected by the optical monitoring system. In this case, the undetected (but real) crack could not be followed immediately. Once the crack grew and spotted, the crack length measurement started. Therefore, a probability of detection $(P O D)$ can be used to evaluate crack the true length. The $P O D$ can be defined as the probability that the optical monitoring used in this study will detect a crack of true length a, which is denoted by $P O D(a)$.

The POD curve for the optical crack detection method used in this study was obtained by reviewing observation of cracks of various lengths at different experiments, and by calculating the ratio of the number of experiments in which the crack of the specified length was detected to the total number of experiments. Generally, $P O D$ increases with crack length and eventually attains a maximum value of unity at which all the cracks will be detectable.

The $P O D$ for various crack lengths was calculated for the lab environment based on the hit-or-miss concept [33] as the ratio of the number of successful detections of a particular crack length over the total number of tests. These $P O D$ values are for discrete crack lengths. In order to obtain a continuous $P O D$ curve, the $P O D$ was estimated by a logistic function for this data [33]. The cumulative distribution function of the log-logistic distribution is:

$$
F(a, m, s)=\frac{\exp \frac{\pi}{\sqrt{3}}\left[\frac{\log a-m}{s}\right]}{1+\exp \frac{\pi}{\sqrt{3}}\left[\frac{\log a-m}{s}\right]} \quad\{a, m, s \text { unit: } \mu m\}
$$

where $m$ and $s$ are the parameters of the model and a denotes the crack length. While the model in Equation 1 is general, the specific parameter values should be specified for the specific structure under study. Figure 6 illustrates the POD curve and the fitted logistic cumulative distribution function $(C D F)$. The point estimates of the parameters of the logistic distribution model for the lab condition where these tests were carried out are $m \approx 27 \mu m$ and $s \approx 9 \mu m$.

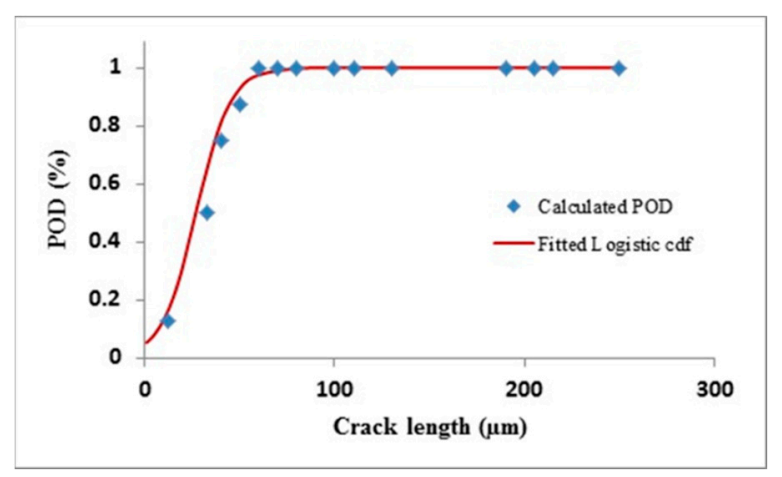

Figure 6. Probability of Detection.

For the consistency of results and practicality of the approach, a minimum detectability limit was used as the initial crack length. This limit of detectability is defined as the smallest crack length for the applied optical microscopy technique with $100 \%$ probability that it will be detected. So, 
$P O D(a)$ would be less than unity for cracks below it. The detectability was selected to be $50 \mu m$. This specified crack length which is smaller than the material's average grain diameter, was used as the subjective point of crack initiation. However, the observed crack lengths with $(a<50 \mu m)$ along with their corresponding PODs $(P O D<1)$ were used in the probabilistic model development. The approach of the probabilistic model development is described in detail in Section 0.

\subsubsection{Crack Length Measurement Error}

Due to measurement errors, the experimental results are uncertain and do not exactly represent true values. The precision and accuracy of optical measurement tools, as well as the sizing techniques used to analyze and process the captured pictures of crack growth, contribute to measurement errors. This experimental measurement error is quantified by cross-validating the measurement results with known true crack lengths.

To obtain a good estimation of the true crack lengths, a microscopy technique was used. When cracked, the specimen was removed from the MTS load frames and was transferred to a fixed stage microscope, which was capable of microflex photomicrography with a HFX camera. Using this setting, the final crack length when the test was stopped was evaluated. An image processing toolbox was used to capture very high quality pictures of the crack at various magnifications. The length of crack was measured later using the captured photographs. In the next step, the true crack lengths from the optiphot microscope were compared with the images of the various stages of cracking using photograph taken from the crack at the last cyclic loading of each test. Figure 7 illustrates the experimentally measured crack lengths versus true crack lengths.

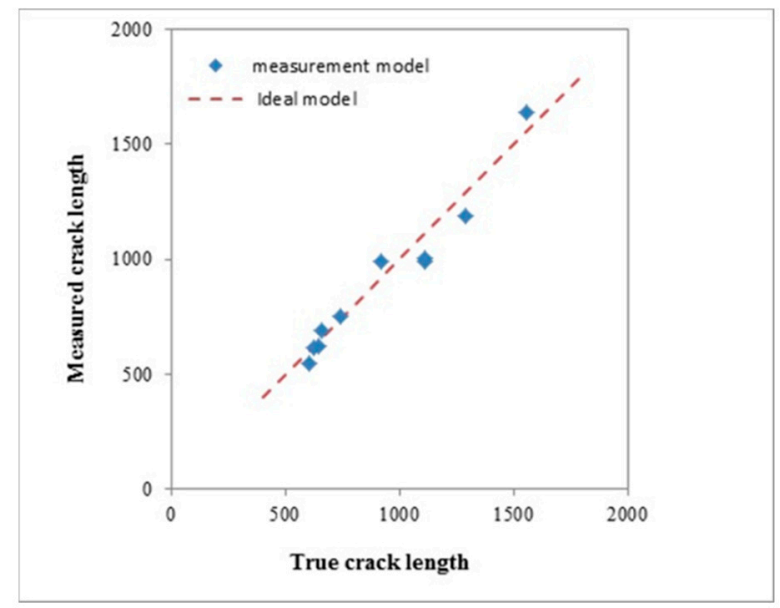

Figure 7. Crack length measurement

Measurement error can then be expressed as a function of measured crack length. To do so, a multiplicative error model described by a lognormal $(L N)$ distribution was used to estimate the measurement error. Based on this methodology, experimentally measured crack lengths are considered to be estimations and representation of the true crack length, given some error as it is shown in Equation 2:

$$
\frac{a_{t, i}}{a_{e, i}}=F_{e, i} ; \quad F_{e} \sim L N\left(b_{e}, s_{e}\right)
$$

where $a_{t, i}$ is the true value of crack length, $a_{e, i}$ indicates the experimental measurement results and Fe is the multiplicative error (both random and systematic) of experimental measurement with respect to true value. The lognormal distribution representing the uncertainty of the multiplicative error has parameters be which is the mean of experimental measurement error, as well as se which is the standard deviation of the experimental measurement error and both estimated using a Bayesian 
framework. The summary statistics for the marginal posterior pdf of parameters $b_{e}$ and $s e$ as well as the distribution of Fe are presented in Table 2.

Table 2. Estimated parameters of measurement error.

\begin{tabular}{c|lllll}
\hline Parameter & Mean & $\begin{array}{l}\text { Standard } \\
\text { Deviation }\end{array}$ & $2.5 \%$ & Median & $97.5 \%$ \\
\hline be & 0.027 & 0.02678 & -0.02497 & 0.02673 & 0.08048 \\
se & 0.0818 & 0.0232 & 0.05062 & 0.07747 & 0.1389 \\
$F e$ & 1.031 & 0.09282 & 0.8584 & 1.026 & 1.236 \\
\hline
\end{tabular}

The posterior distributions of the parameters are shown in Figure 8.

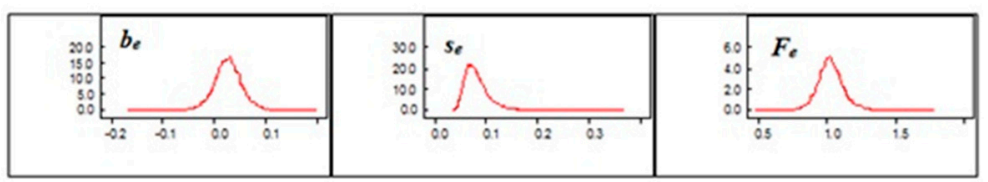

Figure 8. Posterior distribution of multiplicative error of experimental measurement.

Experimental measurement error bounds can be determined from the 2.5 and 97.5 percentile of the multiplicative error of Fe. The resulting upper bound was calculated as $23 \%$ while the lower bound is $-14 \%$. These results are presented graphically in Figure 9. It can be noticed that there is a very slight positive systematic error (bias) from the true value (because the mean $F_{e}$ estimate slightly exceeds unity).

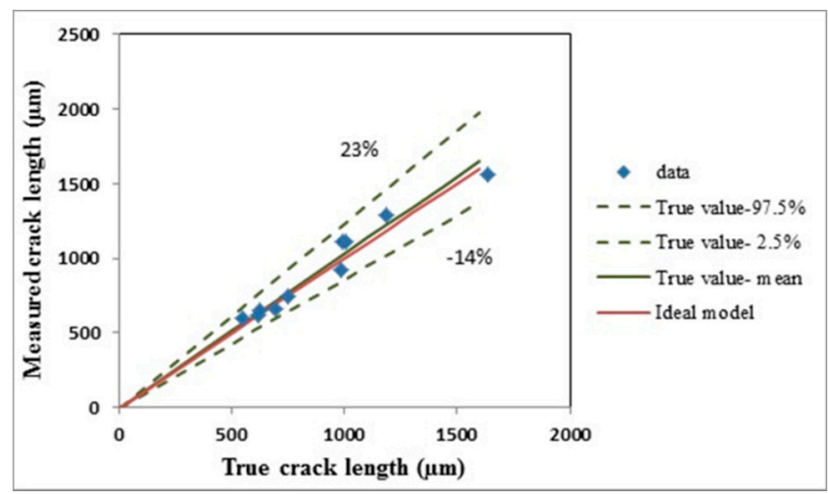

Figure 9. Experimental measurement error.

The estimated parameters of the multiplicative measurement error model listed in Table 2 were used as the prior knowledge of the crack length measurement error in the Bayesian framework provided for the probabilistic model validation approach. The implemented approach considers both uncertainties in the $P O D$ and measurement errors while providing a framework for updating the probability distribution of the model parameters when new data becomes available. For the details of the implemented Bayesian framework refer to section 4.2.

\subsection{Acoustic Emission Results}

Since the primary goal of this study was to develop a method for using AE to monitor small crack growth, it was necessary to find an empirical relation between AE signals and crack length. In order to do this, recorded AE signals were post-processed and the trends in AE events were evaluated for the duration of test. Any association between AE features and crack growth were established and an empirical relation was developed. 


\subsubsection{Acoustic Emission Counts}

After post processing of the recorded AE signals, the number of cumulative counts was calculated and plotted versus loading cycles. Figure 10 illustrates measured cumulative counts for the experiment (T8) at different fatigue loading cycles. A similar trend observed for the measured crack lengths versus number of cycles at the same time window. An example of such a trend is shown in Figure 11.

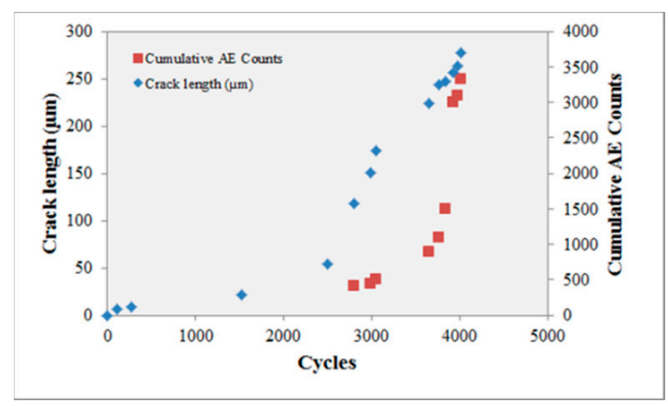

Figure 10. Cumulative AE Counts and Crack length

Results show that cumulative AE counts have considerable correlation with the measured crack lengths. As expected, in all experiments performed the calculated AE cumulative counts showed a linear correlation with the length of crack. Figure 11 clearly shows that the cumulative AE count has a linear relationship with the crack length. A linear model can be developed based on this relation as shown in Figure 11.

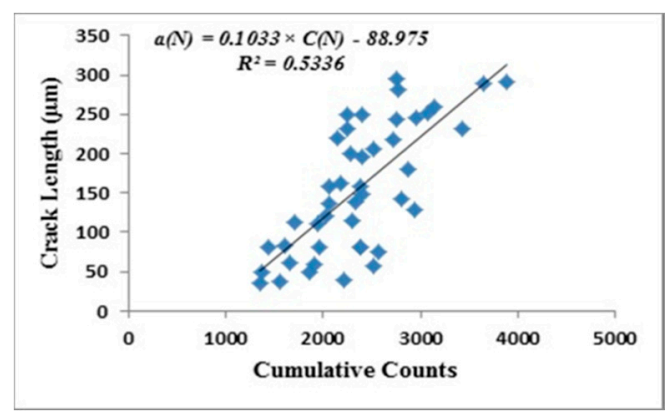

Figure 11. Correlation between Cumulative counts and crack length.

\subsubsection{Acoustic Emission Intensity}

In order to provide a more effective AE measure of damage, a new approach was developed which employs multiple features of the AE signal and provides a measure for strength of signals. The main idea is that larger cracks produce stronger AE signals and not only the total number of counts, but also with the amplitude level of the AE signal, can quantify this strength. Therefore, estimation of small crack length can be implemented by simultaneous evaluation of these AE features. Based on this idea, a multiplicative correction factor was applied on the acquired counts using the observed amplitude of the signal. This correction factor was defined by the ratio of amplitude over the average or benchmark amplitude of signals.

A new AE index called AE-Intensity was proposed to combine AE counts and amplitude for more effective monitoring of damage state. AE-Intensity is a measure of signal strength and it was shown to have a better correlation with crack lengths (see Figure 12). The intensity of AE signals is defined by Equation 3:

$$
\text { AE_Intensity: } I(t)=C(t) \times \frac{A(t)}{A_{0}} \quad \text {, }
$$


where $C(t)$ is the cumulative counts at a specific time $t, A(t)$ indicates the amplitude of the signal and $A_{0}$ is the amplitude threshold which was subjectively selected as $35 d B$ for small crack analyses. This selection was made based on the filtration method applied on the data, which provides a baseline that any signal amplitude can be compared with.

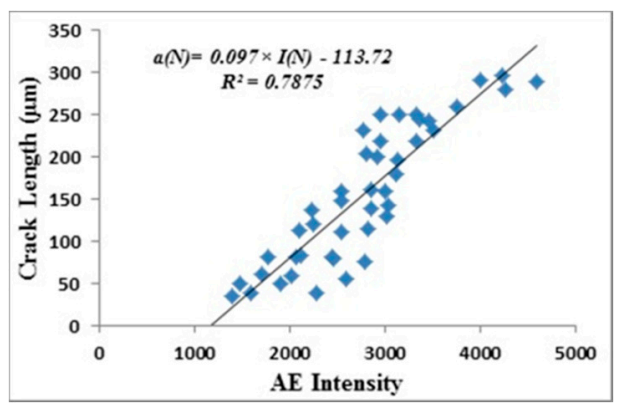

Figure 12. Correlation between corrected counts (intensity) and crack length.

The linear correlation between crack lengths and AE-Intensity is shown in Figure 12. It can be seen that applying the amplitude correction factor to AE count considerably reduces the data scatter in comparison with cumulative counts. Based on the observed relationship, a linear model was proposed which uses AE-intensity as an independent variable and the crack length as dependent variable. The proposed model is introduced in the following section.

\section{Probabilistic Model Development}

The results of the analyzed experimental data were used to develop a probabilistic linear model for the estimation of the small crack length as the dependent variable while AE intensity is considered to be the independent variable. The experimental data obtained in the previous section is divided into two different sets. The first set is used for modeling and the second set is used for estimation of probabilistic error in the model and model validation. The validation data sets were not used in model development (Table 3).

Table 3. Separation of data for Model development

\begin{tabular}{c|c|ccc}
\hline \multirow{7}{*}{} & $\begin{array}{c}\text { Test } \\
\text { Reference }\end{array}$ & $\begin{array}{c}\text { Loading Frequancy } \\
(\mathrm{Hz})\end{array}$ & $\begin{array}{c}\text { Losding Ratio } \\
(\mathrm{R})\end{array}$ & $\begin{array}{c}\text { Force (min-max) } \\
\mathrm{KN}\end{array}$ \\
& T1 & 3 & 0.1 & $0.8-8$ \\
\cline { 2 - 5 } Modeling & T2 & 3 & 0.08 & $0.64-8$ \\
& T3 & 3 & 0.1 & $0.8-8$ \\
& T4 & 2 & 0.5 & $6.5-13$ \\
Validation & T5 & 3 & 0.1 & $0.8-8$ \\
& T6 & 2 & 0.3 & $3-10$ \\
& T7 & 2 & 0.5 & $6.5-13$ \\
& T8 & 2 & 0.3 & $3-10$ \\
\hline
\end{tabular}

\subsection{Modeling}

According to the observed correlation between AE intensity and crack length, a linear relationship was proposed in the following form:

$$
a(N)=\alpha \cdot I(N)+\beta \quad
$$

where $a(N)$ indicates the true length of the small crack after $N$ loading cycles, $I(N)$ is the calculated AE intensity at cycle $N, \alpha$ and $\beta$ are the unknown model parameters. In this analysis, true crack lengths were used to evaluate the parameters of the proposed model. To do so, a MATLAB-based 
routine was developed to implement the measurement uncertainty analysis that was outlined in Section 3.3.

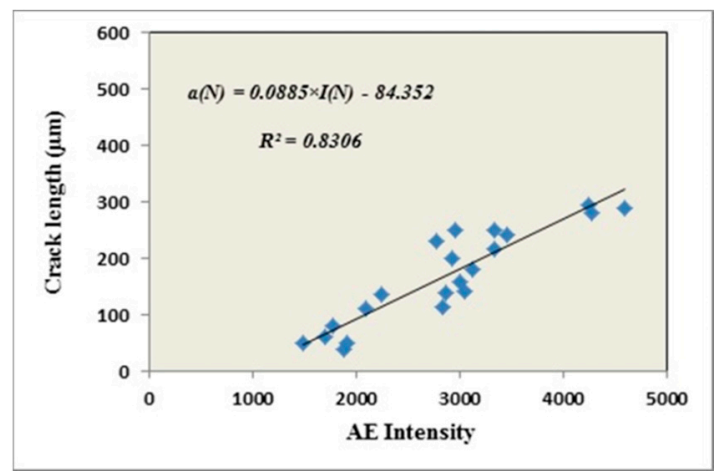

Figure 13. Estimation of Model Parameters.

After the first steps of analyzing the experimental data, results were used to estimate the unknown parameters in the proposed model (Equation 4) using least square regression analysis. Results are shown in Figure 13 and Table 4.

Table 4. Estimated model parameters

\begin{tabular}{cc}
\hline Parameter & Estimated Value \\
\hline$a$ & 0.0885 \\
$\beta$ & -84.352 \\
\hline
\end{tabular}

\subsection{Model Validation and Model Error Estimation}

In order to validate the developed AE model, model predictions of small crack length were compared against the validation experimental data set. For a given value of AE Intensity, a prediction of crack length was estimated based on the developed AE-based model. The prediction results are then compared against the true crack lengths obtained by five validation experiments (listed in Table 3). The Bayesian approach using multiplicative error for model validation was implemented using the validation sets of data to evaluate errors associated with the developed AE-based model and evaluate its prediction uncertainties.

Model validation approach was originally developed by Ontiveros, et al. to account for uncertainties in fire model simulation predictions [34]. In the proposed methodology, both model prediction and experimental results are considered to be estimations and representation of the true values, given some error as it is shown in Equations 2 and 5. The multiplicative error of experimental measurement with respect to the true value was introduced by Equation 2 and the multiplicative error of model prediction with respect to true value is shown in Equation 5:

$$
\frac{\mathrm{a}_{\mathrm{t}, \mathrm{i}}}{\mathrm{a}_{\mathrm{m}, \mathrm{i}}}=\mathrm{F}_{\mathrm{m}, \mathrm{i}} ; \mathrm{F}_{\mathrm{m}} \sim \mathrm{LN}\left(\mathrm{b}_{\mathrm{m}}, \mathrm{s}_{\mathrm{m}}\right)
$$

where $a_{t, i}$ is the true value, $a_{m, i}$ is the model prediction and $F_{m}$ is the multiplicative (fractional) error of the model prediction, with respect to the true value. Parameter $b_{m}$ is the mean (multiplicative) error of the model and $s m$ is the standard deviation of the error. Accordingly, the multiplicative error of the measurement with respect to the model prediction could be defined by Equation 6 :

$$
\frac{\mathrm{a}_{\mathrm{e}, \mathrm{i}}}{\mathrm{a}_{\mathrm{m}, \mathrm{i}}}=\frac{\mathrm{F}_{\mathrm{m}, \mathrm{i}}}{\mathrm{F}_{\mathrm{e}, \mathrm{i}}}=\mathrm{F}_{\mathrm{t}, \mathrm{i}} \quad \text {, }
$$


where $F_{t}$, is the multiplicative error of the model versus measurement. Since both $F_{m, i}$ and $\mathrm{F}_{\mathrm{e}, \mathrm{i}}$ distributions are lognormal, the distribution of $F_{t, i}$ would also be lognormal with mean and standard deviation of $\left(b_{m}-b_{e}\right)$ and $\sqrt{\mathrm{s}_{\mathrm{m}}^{2}+\mathrm{s}_{\mathrm{e}}^{2}}$, respectively.

The proposed Bayesian framework quantifies the uncertainties associated with the POD and crack length measurement error into calculation. The Bayesian approach mathematically combines prior knowledge of crack length with uncertain experimental data and considers the systematic and random measurement errors and associated uncertainties, to estimate the posterior distribution of crack length. For more information about this approach and the concept of Bayesian uncertainty analysis see Ontiveros, et al [34]. In this approach, the combined effect of evidence used: POD data, measurement errors, and measured crack lengths were captured by a likelihood function. Considering Equations 2 and 5, the likelihood of the observed data using combined POD, measurement errors, measured and model-estimated crack can be calculated as:

$$
\begin{aligned}
& L\left(\frac{a_{e, i}}{a_{m, i}}, b_{e}, s_{e} \mid b_{m}, s_{m}, m, s\right) \\
& =\prod_{i=1}^{n}\left(\left(\operatorname{POD}\left(a_{e, i} \mid m, s\right)\right)\left(\frac{1}{\sqrt{2 \pi}\left(\frac{a_{e}, i}{a_{m, i}}\right) \sqrt{s_{m}^{2}+s_{e}^{2}}}\right) \exp \left(\frac{-\left[\ln \left(\frac{a_{e, i}}{a_{m, i}}\right)-\left(b_{m}-b_{e}\right)\right]^{2}}{2\left(s_{m}^{2}+s_{e}^{2}\right)}\right)\right),
\end{aligned}
$$

where, the $P O D$ function is assumed to follow a log-logistic form based on Equation 1:

$$
P O D\left(a_{e, i} \mid m, s\right)=\frac{\exp \frac{\pi}{\sqrt{3}}\left[\frac{\log \left(a_{e, i}\right)-m}{s}\right]}{1+\exp \frac{\pi}{\sqrt{3}}\left[\frac{\log \left(a_{e, i}\right)-m}{s}\right]},
$$

The parameters $b_{e}$ which is the mean of experimental measurement error, as well as se which is the standard deviation of the experimental measurement error were previously estimated as discussed in Section 3.2. The summary statistics for the marginal posterior $p d f$ of parameters $b_{m}$ and $S_{m}$ as well as the distribution of $F_{m}$ are presented in Table 5.

Table 5. Multiplicative error statistic summary

\begin{tabular}{cccccc}
\hline Paramster & Mean & $\begin{array}{l}\text { Standard } \\
\text { Deviation }\end{array}$ & $2.5 \%$ & Median & $97.5 \%$ \\
$b_{n}$ & -0.0575 & 0.02983 & -0.1159 & -0.0575 & 0.00021 \\
$s_{n}$ & 0.2179 & 0.02346 & 0.1765 & 0.2163 & 0.2676 \\
$F_{m}$ & 0.9682 & 0.2188 & 0.6095 & 0.9441 & 1.465 \\
\hline
\end{tabular}

The posterior distributions of the parameters are also shown in Figure 14

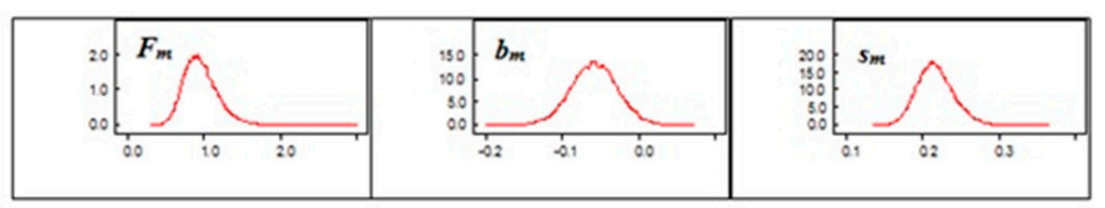

Figure 14. Distribution of multiplicative error and it parameters

Model uncertainty bounds for the crack length estimation can be determined from the 2.5 and 97.5 percentiles of the multiplicative error of $\mathrm{Fm}$. The resulting upper bound was calculated as $46 \%$ while the lower bound is $-39 \%$. These results are presented graphically in Figure 15. It can be noticed that there is a very slight systematic errors (bias) from the true value in the results showing that the model slightly overestimates the true length of the crack. 


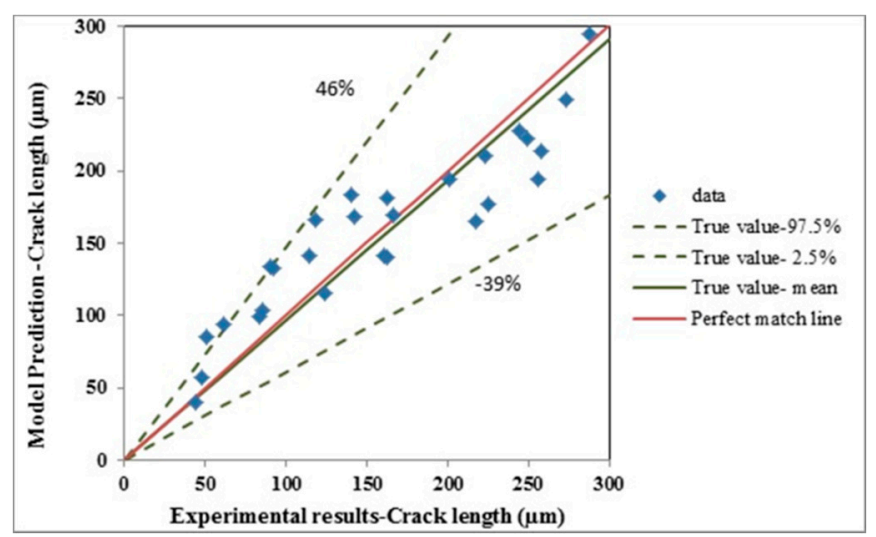

Figure 15. Comparison of AE model prediction and experimental results

Assuming $a_{m}$ is the model prediction of crack length, the true crack length model prediction then can be estimated by multiplying the distribution of am by $F_{m}$ :

$$
\mathrm{a}_{\mathrm{t}}=\mathrm{F}_{\mathrm{m}} \cdot \mathrm{a}_{\mathrm{m}} \quad \text {, }
$$

which can be estimated by a lognormal distribution as Equation 10:

$$
a_{t} \sim L N\left(\ln \left(a_{m}\right)+b_{m}, s_{m}\right)
$$

The model prediction results were modified using the resulted bias distribution. Figure 15 illustrates the model prediction uncertainty bounds as well as the modified prediction results. As it can be seen, the developed model slightly over predicts the crack lengths.

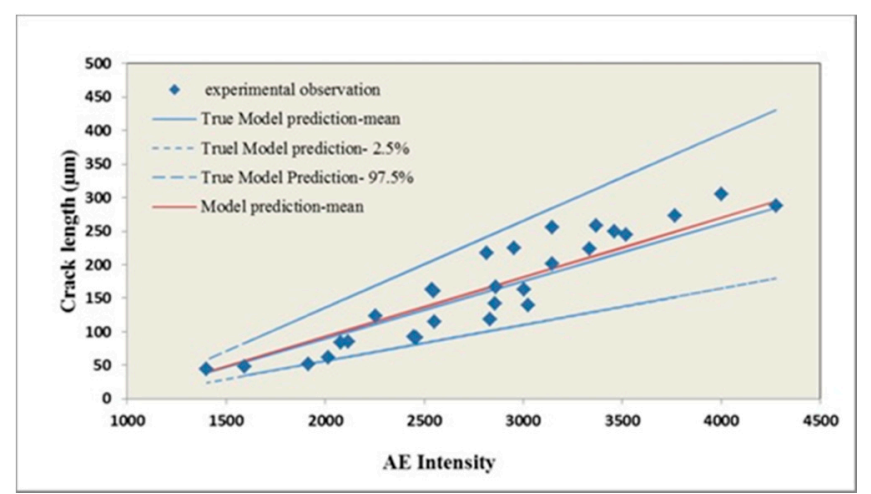

Figure 16. Model Prediction with multiplicative error

The results in Figure 15 show that the developed AE-model reasonably estimates the true crack length. There is a small bias in prediction that can be accounted for with the inclusion of the model error factor, $F_{m}$, which allows for estimation of the distribution that describes the true crack length. The resulting model can be used to appropriately estimate the true crack length distribution, without a bias. By using the developed model, for a given value of $I(N)$, distribution of crack length can be estimated. The result of the posterior predictive distribution for $a(N)$ as a function of $I(N)$ is plotted in Figure 16.

\section{Conclusions}

A novel methodology for in-situ monitoring of small fatigue crack initiation and growth using $\mathrm{AE}$ signal processing technique was introduced. Investigation of the uniform cyclic loading tests on Al7075-T6 specimens indicated that initiation of cracks smaller than the grain size could be identified using statistical analysis of the resulting AE signals. Several standard fatigue tests were 
performed using flat dog-bone specimens. Acoustic Emission data acquisition was used in conjunction with optical microscopy for online monitoring of crack length. The data captured was used to establish a correlation between certain AE signal features and the measured crack length. Also a probabilistic model of fatigue crack length distribution based on a combination of $\mathrm{AE}$ signal characteristics including probability of crack detection and measurement error was developed. The proposed model was validated and proved to be effective for detection of crack initiation as well as prediction of small crack growth in early stages of propagation. Development of the proposed AE monitoring technique reported in this paper facilitates early detection of fatigue crack, allows for the prognostics and life predictions of the structure.

Acknowledgments: This work was partially sponsored by the U.S. Navy's NAVAIR under the cooperative agreement N0042198H1116.

Conflicts of Interest: The authors declare no conflict of interest.

\section{References}

1. Forth, S.C.; Newman, J.C.; Forman, R.G. Evaluation of Fatigue Crack Thresholds Using Various Experimental Methods. J. of ASTM Int. 2005, 2, 1-16.

2. Künkler, B.; Düber, O.; Köster, P.; Krupp, U.; Fritzen, C-P.; Christ, H.-J. Modelling of short crack propagation - Transition from stage I to stage II. Eng. Frac. Mech. 2008, 75, 715-725.

3. McDowell, D.L. An engineering model for propagationof small cracks in fatigue. Eng. Frac. Mech. 1997, $56,357-377$.

4. Shyam, A.; Allison, J.; Jones, J. A small fatigue crack growth relationship and its application to cast aluminum. Acta Materialia 2005 53,1499-1509.

5. Roberts, T.M.; Talebzadeh, M. Fatigue life prediction based on crack propagation and acoustic emission count rates. J. of Constr. Steel Research 2003, 59, 679-694.

6. Rabiei, M.; Modarres, M. Quantitative methods for structural health management using in situ acoustic emission monitoring. Int. J. of Fatigue $2013,49,81-89$.

7. Keshtgar, A.; Modarress, M. Acoustic Emission-Based Fatigue Crack Growth Prediction, Proceeding of Reliability and Maintainability Symposium (RAMS), Orlando, U.S.A. 2013.

8. Marquez, J.G.M.; Olivares, J. L. A study of crack initiation and propagation in Ni-Cr thermally sprayed coatings using Acoustic Emission techniques. Thin Solid Films 1987, 153, 243-252.

9. Chaswal, V.; Sasikala, G.; Ray, S.K.; Mannan, S.L.; Raj, B. Fatigue crack growth mechanism in aged 9Cr-1Mo steel: threshold and Paris regimes. Mat. Sci. \& Eng. 2005, 395, 251-264.

10. Rahman, Z.; Ohba, H.; Yoshioka, T.; Yamamoto, T. Incipient damage detection and its propagation monitoring of rolling contact fatigue by acoustic emission. Tribology Int. 2009, 42, 807-815.

11. Elforjani, M.; Mba, D. Natural mechanical degredation measurements in slow speed bearings. Eng Failure Analysis 2009, 16, 521-532.

12. Han Z.; Luo, H.; Sun, C.; Li, J.; Papaelias, M.;Davis, C. Acoustic emission study of fatigue crack propagation in extruded AZ31 magnesium alloy. Materials Science \& Engineering 2014, 597, 270-278.

13. Capek J.; Mathis, K.; Clausen, B.; Straska, J.; Beran, P.; Lukas, P. Study of the loading mode dependence of the twinning in random textured cast magnesium by acoustic emission and neutron diffraction methods. Mat. Sci. \& Eng. 2014, 602, 25-32.

14. Vanniamparambil, P.A.; Guclu, U.; Kontsos A. Identification of crack initiation in aluminum alloys using acoustic emission. Experimental Mechanics 2015, 55, 837-850.

15. Mazal, P.; Vlasic, F.; Koula, V. Use of acoustic emission method for identification of fatigue microcracks creation. Procedia Engineering 2015,133, 379-388.

16. Beattie, A.G. Acoustic emission, principles and instrumentation. J. of Acoustic Emission 1983, 2, 95-128.

17. Berkovits, D.; Fang, D. Study of fatigue crack characteristics by acoustic emission. Eng. Frac. Mech. 1995, 51, 401-416.

18. Eberhardt, E.; Stead, D.; Stimpson, B.; Read, R.S. Changes in Acoustic event properties with progressive fracture damage. Int. J. of Rock Mech. \& Min. Sci. 1997, 34, 3-4.

19. Miller, R.K.; Hill, E.V.K.; Moore, P.O. In Nondestructive Testing Handbook, 3rd ed.; 2005; Volume 6.

20. Physical Acoustic Corporation, AEwin Software User's Manual, Princeton Junction, NJ, 2007. 
21. Kappatos, V.; Dermatas, E. Crack detection in noisy environment including raining conditions. Aircraft Eng. E Aerospace Tech. 2007, 79, 163-169.

22. Larsen J.M.; Allison,J.E. Small Crack Test Methods. American Society for Testing and Materials: Philadelphia, PA, USA, 1992.

23. Anderson,T. Fracture Mechanics: Fundamentals and Applications. Boca Raton: Boca CRC Press, 1995.

24. Papazian ,J.M.; Anagnostou, E.L.; Engel, S.J.; Hoitsma, D.; Madsen, J.; Silberstein, R.P.; Welsh, G.; Whiteside, J.B. A structural integrity prognosis system. Engineering Fracture Mechanics 2009, 76, 620632.

25. Iyyer, N.; Sarkar, S.; Merrill, R.; Phan, N. Aircraft life management using crack initiation and crack growth models - P-3C Aircraft experience. Int. J. of Fatigue, 2007, 29, 1584-1607.

26. Bhattacharya B.; Ellingwood, B.; Continum damage mechanics analysis of fatigue crack initiation. J. of Fatigue 1998, 20, 631-639.

27. Pearson, S.; Initiation of fatigue cracks in commercial aluminium alloys and the subsequent propagation of very short cracks, Eng. Frac. Mech. 1975, 7, 235-247.

28. Xue, Y.; McDowell, D.L.; Horstemeyer, M.F.; Dale, M.H.; Jordan, J.B. Microstructure-based multistage fatigue modeling of aluminum alloy 7075-T651. Eng. Frac. Mech. 2007, 74, 2810-23.

29. ASTM E466 : Standard practice for coducting force controlled constant amplitude axial fatigue tests of metallic materials, ASTM International, 2012; Volume i.

30. Morton, T.M.; Harrington, R.M.; Bjeletich, J.G. Acoustic Emission of fatigue crack growth. Eng. Frac. Mech. 1973, 5, 691-697.

31. Wang, Z.F.; Li, J.; Ke, W.; Zhu, Z. Acoustic emission monitoring of fatigue crack closure. Scripta Metallurgica et Materialia 1992, 27, 1691-1694.

32. Roberts, T.M.; Talebzadeh, M. Acoustic emission monitoring of fatigue crack propagation. J. of Constructional Steel Research 2003, 59, 695-712.

33. Georgiou, G.A. Probability of Detection ( PoD ) curves: Derivation, applications and limitations, research report: Jacobi Consulting Limited, 2006, London.

34. Ontiveros, V.; Cartillier, A.; Modarres, M. An Integrated Methodology for Assessing Fire Simulation Code Uncertainty. Nuclear Sci. \& Eng. 2010, 166, 179-201.

(C) 2017 by the authors; licensee Preprints, Basel, Switzerland. This article is an open access article distributed under the terms and conditions of the Creative Commons by Attribution (CC-BY) license (http://creativecommons.org/licenses/by/4.0/). 